\title{
Breast Mass Characterization Using 3-Dimensional Automated Ultrasound as an Adjunct to Digital Breast Tomosynthesis
}

\author{
A Pilot Study
}

Frederic Padilla, PhD, Marilyn A. Roubidoux, MD, Chintana Paramagul, MD, Sumedha P. Sinha, PhD, Mitchell M. Goodsitt, PhD, Gerald L. Le Carpentier, PhD, Heang-Ping Chan, PhD, Lubomir M. Hadjiiski, PhD, J. Brian Fowlkes, PhD, Annette D. Joe, MD, Katherine A. Klein, MD, Alexis V. Nees, MD, Mitra Noroozian, MD, Stephanie K. Patterson, MD, Renee W. Pinsky, MD, Fong Ming Hooi, MS, Paul L. Carson, PhD

Received March 22, 2012, from the Department of Radiology, University of Michigan Medical Center, Ann Arbor, Michigan USA. Revision requested May 1, 2012. Revised manuscript accepted for publication June 14, 2012.

We thank Mark Helvie, Chris Lashbrook, Lisa DeCaussin, Jeanne Hill, Becca Booi, PhD (currently with Sg2, Inc, Chicago, IL), and Ganesh Narayanasamy, PhD (currently with University of Kentucky, Lexington, KY), all from the Department of Radiology at the University of Michigan; Anne Hall, PhD, of GE Healthcare (Milwaukee, WI); Jeff Eberhard, PhD, and Andrea Schmitz, MS, for system development; Kai Thomenius, PhD, Carl Chalek, MS, and numerous other staff and scientists at GE Global Research (Niskayuna, NY); and Lorenzo Pesce, PhD, from the Department of Radiology at the University of Chicago, for fruitful discussions about receiver operating characteristic curve statistics. This study used a digital breast tomosynthesis unit prototype and an initial automated ultrasound system developed by General Electric Global Research with the support of the University of Michigan under National Institutes of Health grant RO1 CA 091713 and the Uniformed Services University of the Health Sciences under Office of Naval Research grant MDA9050210012.

Address correspondence to Frederic Padilla, $\mathrm{PhD}$, Department of Radiology, University of Michigan Medical Center, 3218A Medical Science Building I, 1301 Catherine St, Ann Arbor, MI 48109 USA.

E-mail:fpadilla@umich.edu

Abbreviations

AUS, automated ultrasound; $A_{z}$, area under the curve; BI-RADS, Breast Imaging Reporting and Data System; DBT, digital breast tomosynthesis; $R O C$, receiver operating characteristic; 3D, 3-dimensional; US, ultrasound
Objectives-The purpose of this study was to retrospectively evaluate the effect of 3-dimensional automated ultrasound (3D-AUS) as an adjunct to digital breast tomosynthesis (DBT) on radiologists' performance and confidence in discriminating malignant and benign breast masses.

Methods - Two-view DBT (craniocaudal and mediolateral oblique or lateral) and singleview $3 \mathrm{D}$-AUS images were acquired from 51 patients with subsequently biopsy-proven masses (13 malignant and 38 benign). Six experienced radiologists rated, on a 13-point scale, the likelihood of malignancy of an identified mass, first by reading the DBT images alone, followed immediately by reading the DBT images with automatically coregistered 3D-AUS images. The diagnostic performance of each method was measured using receiver operating characteristic (ROC) curve analysis and changes in sensitivity and specificity with the $\mathrm{McNemar}$ test. After each reading, radiologists took a survey to rate their confidence level in using DBT alone versus combined DBT/3D-AUS as potential screening modalities.

Results - The 6 radiologists had an average area under the ROC curve of 0.92 for both modalities (range, 0.89-0.97 for DBT and 0.90-0.94 for DBT/3D-AUS). With a Breast Imaging Reporting and Data System rating of 4 as the threshold for biopsy recommendation, the average sensitivity of the radiologists increased from $96 \%$ to $100 \%(P>.08)$ with 3D-AUS, whereas the specificity decreased from $33 \%$ to $25 \%(P>.28)$. Survey responses indicated increased confidence in potentially using DBT for screening when 3D-AUS was added $(P<.05$ for each reader).

Conclusions - In this initial reader study, no significant difference in ROC performance was found with the addition of 3D-AUS to DBT. However, a trend to improved discrimination of malignancy was observed when adding 3D-AUS. Radiologists' confidence also improved with DBT/3DAUS compared to DBT alone.

Key Words - automated ultrasound; breast; reader study; tomosynthesis

U ltrasound (US) is essential for characterization of breast masses. ${ }^{1}$ It is commonly used as an adjunct to diagnostic clinical mammography, the standard imaging method for detecting breast cancer. The interpretation of handheld US images by experienced radiologists leads to accuracy close to $100 \%$ for dif- 
ferentiating simple cysts from other lesions ${ }^{2}$ and can improve distinction of malignant from benign breast masses. ${ }^{3-5}$

Three-dimensional automated ultrasound (3D-AUS) scanners are currently being investigated ${ }^{6-15}$ as a means to reduce the breast US examination time, measure additional tissue properties such as the speed of sound and acoustic attenuation, ${ }^{16}$ and provide $3 \mathrm{D}$ volumes that can be reviewed after the examination. When compared to handheld US, 3D-AUS has been found to provide similar image quality and diagnostic information, ${ }^{13}$ Breast Imaging Reporting and Data System (BI-RADS) ratings, ${ }^{7,12,13}$ and mass detection rates. ${ }^{7}$ As an adjunct to mammography, 3D-AUS improved mass characterization, ${ }^{17}$ and in a recent screening study, ${ }^{6,18} 3 \mathrm{D}$-AUS improved cancer detection and accuracy and reduced callback rates.

We have used a combined digital breast tomosynthesis (DBT) and 3D-AUS imaging system, developed by GE Global Research (Niskayuna, NY) with our collaboration and modifications. The breast is imaged sequentially with DBT and 3D-AUS under the same mammographic compression using a special dual-modality compression paddle. Imaging in the same geometry ${ }^{8-11}$ provides coregistration between the two modalities. ${ }^{8}$ Coregistration potentially reduced the potential for misidentification of the location of abnormalities as can occur when handheld US is combined with mammography because the two modalities are performed in different geometries. ${ }^{19,20}$

Digital breast tomosynthesis is of interest for breast screening and diagnosis because of its 3D imaging capabilities and its potential to alleviate a limitation of mammography, the masking of noncalcified cancers by other dense tissue. ${ }^{21}$ Digital breast tomosynthesis has shown clinical potential compared to screen-film and digital mammography ${ }^{21}$ by subjectively providing better image quality $22-25$ and increasing the number of detected abnormalities ${ }^{23,26-28}$ and the accuracy in classification of masses. ${ }^{22,29,30}$ In clinical practice, its sensitivity was found to be similar to the sensitivity of mammography. ${ }^{31}$

Whether US, and in particular 3D-AUS, could be as useful an adjunct to DBT for masses as it is for mammography currently in diagnostic evaluations has not been substantially investigated. We report here the results from an initial reader study. Our purposes were to preliminary evaluate the effect of adding 3D-AUS to DBT on radiologists' abilities to discriminate malignant and benign breast masses, to assess radiologists' abilities to detect lesions on 3D-AUS images using coregistration, and to measure radiologists' confidence in using combined DBT/3DAUS as an indicator of its utility in screening and diagnostic breast imaging.

\section{Materials and Methods}

\section{Data Set}

Institutional Review Board approval was granted for this investigation. Informed consent, including consent for future retrospective data analysis, was obtained for each patient. The study was compliant with the Health Insurance Portability and Accountability Act.

Ninety-four patients, each with a mass assessed as suspicious or highly suggestive of malignancy (BI-RADS 4 and 5) based on clinical diagnostic mammography and US imaging, were recruited between 2006 and 2009. All underwent DBT and 3D-AUS research scans before biopsy or fine-needle aspiration. Inclusion criteria for the reader study were a visible mass on DBT, a mass in the field of view of the 3D-AUS image, and availability of pathologic results. Forty-three patients were excluded for the following reasons: technical problems during 3D-AUS acquisition (7), aborted biopsy (7), negative DBT findings (8), and exclusion of the mass from the field of view for 3D-AUS (21). The 7 cases excluded for technical problems were excluded because of communication issues between the main computer and the US scanner, resulting in asynchronous triggering signals and random numbers of slices in the US data sets. The last case was excluded because of an issue during the DBT acquisition, resulting in poor image quality. Both issues took place in the early stage of the study and were subsequently corrected.

The final case set thus consisted of 51 patients (mean age 50 years; range, 29-79 years) for a total of 51 masses (mean diameter, $1.8 \pm 1.1 \mathrm{~cm}$ ). Thirteen masses were malignant (10 invasive ductal carcinomas, 2 invasive lobular carcinomas, and 1 metaplastic carcinoma), and 38 were benign ( 13 cysts, 12 fibroadenomas, 2 papillomas, 1 lobular carcinoma in situ, and 10 other benign breast diagnoses). In 2 of the 51 cases, the 3D-AUS findings were negative, as were the clinical handheld US findings.

\section{Imaging Procedures and Equipment}

Craniocaudal and either lateral or mediolateral oblique DBT images were obtained with the use of a DBT unit prototype ${ }^{8}$ with the patient in a seated position. Breast compression was applied, similar to but often slightly reduced from that of mammography. Twenty-one projection views were acquired in 7.5 seconds with a total mean glandular dose of approximately 1.4 times that for a conventional screen-film mammogram as estimated with an American College of Radiology phantom. Image reconstruction was performed with a simultaneous algebraic reconstruction technique ${ }^{32}$ for a pixel size the same as the 
detector element pitch $(0.1 \times 0.1 \mathrm{~mm})$ and slice spacing set to $1 \mathrm{~mm}$.

After the second DBT view, a single automated whole-breast US scan was acquired in the same view during the same compression, ${ }^{10}$ using either a solid plastic polymethylpentene $\mathrm{e}^{10,11,33,34}$ or, in a few cases, a fiber mesh $^{35}$ compression paddle, with the contact surface of the breast and the proximal breast periphery covered by US coupling gel. A linear matrix array US transducer (GE $\mathrm{M} 12 \mathrm{~L}$ ) operated at $10 \mathrm{MHz}$ was translated across the compression paddle with a computer-driven motorized transducer carriage. The image plane of the transducer was perpendicular to the chest wall, whereas the motion of the carriage was parallel. Images were acquired with a LOGIQ 9 US system (GE Healthcare, Milwaukee, WI). Image spacing of $0.4 \mathrm{~mm}$ was achieved using an external image frame trigger that was developed for the LOGIQ 9 system.

\section{Visualization Software}

In-house-developed software was used to display DBT and 3D-AUS images. The tomosynthesis slices were displayed on a T221 9-megapixel, 22.2-in-diagonal liquid crystal display monitor (IBM, Armonk NY), and the 3D-AUS slices were displayed on an adjacent 2-megapixel, 24-indiagonal monitor. Both the DBT and 3D-AUS images were displayed with the original pixel resolution without subsampling. Readers could adjust contrast, brightness, and zoom and scroll through the slices for viewing. The software performed automatic registration of the lesion location from the DBT to the 3D-AUS volumes. The accuracy of the registration was estimated to be $0.8 \pm 0.3 \mathrm{~mm}$ for a rigid calibration phantom. ${ }^{8}$ For patient data, a spatial discrepancy of up to $5 \mathrm{~mm}$ in the registration was noticed due to usual patient movements, a tendency of the patients to pull back over the several minutes of compression, and variations in the speed of sound in the breast and refraction of the US beam.

\section{Mass Localization}

The true positions of the biopsied masses on the DBT and 3D-AUS volumes were established by a Mammography Quality Standards Act-qualified radiologist (C.P.), who had access to clinical images (mammography and handheld US) and clinical information. An independent verification was performed by a second qualified radiologist (M.A.R.).

\section{Reader Study Design}

Six academic breast radiologists (A.D.J., K.A.K., A.V.N., M.N., S.K.P., and R.W.P.), with 3 to 20 (median, 13) years of experience in mammographic and breast US interpre- tation, participated as observers. All were Mammography Quality Standards Act qualified, and 5 were fellowship trained in breast imaging. These readers read the cases in randomized order during an average of 3 reading sessions. The radiologists were not informed about the cancer prevalence in the data set, and the results of their assessments were not discussed with them before the entire study was completed.

In the 2-step sequential reading design used, the radiologists first examined the images of the mass in the craniocadal and mediolateral oblique (or lateral) DBT views. The position of the mass in these volumes was marked by a region of interest. The radiologists first categorized the masses from 1 to 5 using the American College of Radiology's BI-RADS classification system. Category 0 (additional imaging needed) was not allowed. The likelihoods of malignancy were also rated using a 13-point scale, with 1 being normal; 2 , benign; 3 , probably benign with a $0 \%$ to $2 \%$ risk of malignancy; 4 , suspicious with a $3 \%$ to $10 \%$ risk; 5 , suspicious with an $11 \%$ to $20 \%$ risk; and up to 13 , highly suggestive of malignancy with a risk higher than $94 \%$. Readers were reminded at the beginning of each session that a rating of greater than $2 \%$ likelihood of malignancy (BI-RADS 4 or 5) implied a biopsy recommendation. The radiologists then selected descriptors of the shape, margin, and mass density, according to the BI-RADS system. The presence of absence of calcifications was recorded. All assessments were recorded with an interactive graphical user interface.

Immediately after reading the DBT images for a case, the radiologists examined the 3D-AUS volume corresponding to the last DBT view for the case while the DBT volumes were still displayed. This mode is referred to as DBT/3D-AUS below. The region of interest of the mass in the DBT volumes was coregistered and displayed on the AUS volume. The radiologists were informed of the potential imprecision of the coregistration. They first localized the position of the mass on the 3D-AUS images by marking a region of interest. They then selected descriptors from a list of terms in the American College of Radiology's BI-RADS US lexicon to characterize the mass shape, orientation, margin, echo pattern, posterior acoustic features, lesion boundary, and surrounding tissues. They finally provided a BI-RADS classification and a likelihood of malignancy rating using combined information retrieved from DBT and 3D-AUS.

After each reading of DBT and combined DBT/3DAUS volumes, the radiologists rated their confidence in using DBT and combined DBT/3D-AUS for simultaneous screening and diagnostic examinations. The sur- 
vey question that was asked after each DBT reading was: "If screening were performed by tomosynthesis in this fashion, would tomosynthesis alone allow you to avoid categorizing this case as BIRADS 0 and instead enable you to categorize it as BIRADS 2, 3, 4, or 5, without any additional imaging? (Assume no previous comparison mammograms.)" The survey question that was asked after each combined DBT/3D-AUS reading was: "If screening were performed by combined tomosynthesis and US as we presented, are these images sufficient to allow you to avoid categorizing this case as BIRADS 0 and instead enable you to categorize it as BIRADS 2, 3, 4, or 5 without any additional imaging? (Assume no previous comparison mammograms.)" Answers were recorded using a 10-point scale, 1 being "no," 5 being "maybe," and 10 being "yes."

Because the main objective of the study was to preliminary evaluate the effect of adding 3D-AUS to DBT on radiologists' abilities to discriminate malignant and benign breast masses, it was necessary to have the reading of the mass of interest on both modalities for all the radiologists. To make sure that the correct masses were detected on the 3D-AUS images, 2 reference radiologists reviewed the mass localizations on the 3D-AUS images for each reader. The cases with incorrectly identified masses of interest on the 3D-AUS images were identified. The correct areas of the masses of interest were then marked on the 3D-AUS images. At least 2 months after the readers' reading session, the 5 readers who had incorrectly identified the locations of 1 or more masses of interest on the 3D-AUS images were recalled to again read those cases. For the second readings, the masses of interest were already marked on the 3D-AUS images. For cases in which there were such second readings, the ratings for the second readings replaced the ratings for the first readings in the analyses of the readers' performances.

\section{Data and Statistical Analyses}

The observer performances in terms of likelihood of malignancy ratings were analyzed using the receiver operating characteristic (ROC) method. ${ }^{36,37}$ The statistical significance of the difference in the area under the ROC curve $\left(A_{z}\right)$ between the two modalities was tested using the DorfmanBerbaum-Metz multireader multicase method 38,39 (http:// www-radiology.uchicago.edu/krl/KRL_ROC/ software_index6.htm). This method takes into account both the reader and the case sample variations by means of an analysis of variance approach. The results can thus be generalized to the population of readers and to the population of samples.

To analyze changes in sensitivity and specificity, cases were classified as "no biopsy recommended" (BI-RADS ratings 1-3) or as "biopsy recommended" (BI-RADS ratings 4 and 5). The individual change in sensitivity for a given radiologist with 3D-AUS was investigated using the McNemar test (WinStat version 2005.1; R. Fitch Software, Lehigh Valley, PA) with consideration of the number of beneficial and detrimental changes in biopsy recommendations for malignant masses. For a malignant mass, a change from no biopsy with DBT to biopsy with combined DBT/3D-AUS was defined as beneficial, and a change from biopsy to no biopsy was defined as detrimental. The McNemar test was similarly applied to the benign masses to assess changes in specificity. Finally, the change in the average ratings for the two survey questions was assessed using the Student 2-tailed paired $t$ test.

\section{Results}

\section{Receiver Operating Characteristic Curve Analysis}

The average $\mathrm{A}_{\mathrm{z}}$ for the likelihood of malignancy across the 6 readers was 0.92 for both DBT alone (range, $0.89-0.97$ ) and combined DBT/3D-AUS (range, 0.90-0.94; Table 1 and Figure 1). The $A_{z}$ values increased for 3 radiologists and decreased for 2 others, but these changes did not achieve statistical significance.

\section{Sensitivity, Specificity, and Changes in Biopsy \\ Recommendations}

With a BI-RADS rating of 4 as the threshold for biopsy recommendation, the average sensitivity of radiologists increased from $96 \%$ for DBT alone to $100 \%$ for combined DBT/3-D AUS, whereas the specificity decreased from $33 \%$ to $25 \%$ (Table 2). With the addition of 3D-AUS, 3 readers upgraded the same malignant mass to BI-RADS 4 or higher, and all malignant masses were correctly diagnosed by all 6 radiologists. The changes in sensitivity, speci-

Table 1. Area Under the Curve Values for 6 Radiologists in Characterization of Masses on DBT and DBT/3D-AUS

\begin{tabular}{lccc}
\hline & \multicolumn{3}{c}{$\mathbf{A}_{\mathbf{z}}$} \\
\cline { 2 - 4 } Radiologist & $\mathbf{D B T}$ & $\mathbf{D B T} / 3 \mathbf{D}-\mathbf{A U S}$ & $\boldsymbol{P}^{\mathbf{a}}$ \\
\hline 1 & 0.92 & 0.93 & .67 \\
2 & 0.91 & 0.91 & .88 \\
3 & 0.93 & 0.94 & .77 \\
4 & 0.89 & 0.90 & .78 \\
5 & 0.92 & 0.91 & .76 \\
6 & 0.97 & 0.94 & .29 \\
Average & 0.92 & 0.92 & .89 \\
\hline
\end{tabular}

${ }^{a}$ The significance of the change in the $A_{z}$ value for the group of radiologists and for each radiologist was estimated by the DorfmanBerbaum-Metz method. 


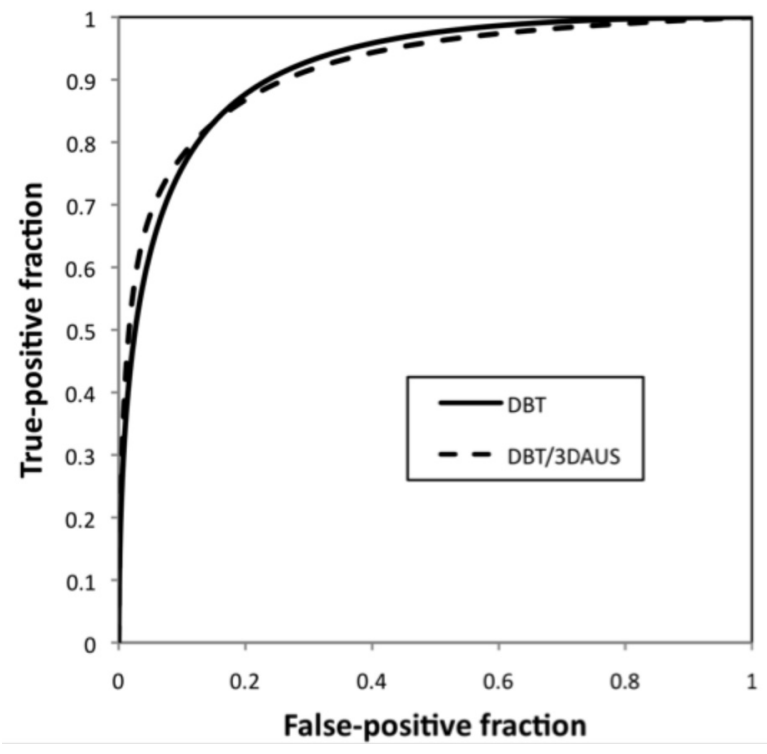

Figure 1. Receiver operating characteristic curves for DBT alone and combined DBT/3D-AUS. The curves were constructed by taking the median of the true-positive fraction among the readers for each value of the false-positive fraction. ficity, and biopsy recommendations did not achieve statistical significance ( $\mathrm{McNemar}$ test, $P>.99$ for sensitivity; $P=.13-.55$ for specificity), except for radiologist 3 , for whom the decrease in specificity was significant $(P<.05$, $\mathrm{McNemar}$ test). On average per radiologist, the number of correct biopsy recommendations per reader (Table 3) increased by 0.5 per 51 cases, and the number of incorrect biopsy recommendations increased by 3.2 per 51 cases. Our study included a case of lobular carcinoma in situ, categorized as benign. This mass was recommended for biopsy by 4 readers with DBT and by 3 readers with DBT/3D-AUS.

\section{Cyst Cases}

The changes in biopsy recommendations were analyzed specifically for the subgroup of cyst cases. On average across the 6 readers, the number of biopsy recommendations for the 13 cyst cases decreased from 6.7 for DBT to 5.3 for combined DBT/3D-AUS (Tables 4 and 5). This decrease was associated with a decrease in the average BI-RADS ratings per case, from 3.49 to $3.10(P<.1$, Student 2-tailed paired $t$ test).

Table 2. Sensitivity and Specificity for Each Radiologist at a Decision Threshold of BI-RADS 4

\begin{tabular}{lcccc}
\hline \multirow{2}{*}{ Radiologist } & \multicolumn{2}{c}{ Sensitivity } & \multicolumn{2}{c}{ Specificity } \\
\cline { 2 - 5 } & \multicolumn{1}{c}{ DBT } & DBT/3D-AUS & DBT & DBT/3D-AUS \\
\hline 1 & $0.92(12)$ & $1.00(13)$ & $0.55(21)$ & $0.47(18)$ \\
2 & $0.92(12)$ & $1.00(13)$ & $0.55(21)$ & $0.18(7)^{\mathrm{a}}$ \\
3 & $1.00(13)$ & $1.00(13)$ & $0.45(17)$ & $0.26(10)$ \\
4 & $0.92(12)$ & $1.00(13)$ & $0.29(11)$ & $0.21(8)$ \\
5 & $1.00(13)$ & $1.00(13)$ & $0.00(0)$ & $0.11(4)$ \\
6 & $1.00(13)$ & $1.00(13)$ & $0.16(6)$ & $0.26(10)$ \\
Average & $0.96(12.5)$ & $1.00(13)$ & $0.33(12.7)$ & $0.25(9.5)$ \\
\hline
\end{tabular}

The significance of the change in sensitivity and specificity for individual readers was estimated by the McNemar test. Data in parentheses are the numbers of correctly classified lesions, ie, true-positive in the Sensitivity column and true-negative in the Specificity column. The total numbers of malignant and benign lesions are 13 and 38, respectively.

a $p<.01$

Table 3. Number of Biopsy Recommendations at a Decision Threshold of BI-RADS 4

\begin{tabular}{|c|c|c|c|c|c|c|}
\hline \multirow[b]{2}{*}{ Radiologist } & \multicolumn{3}{|c|}{ DBT } & \multicolumn{3}{|c|}{ DBT/3D-AUS } \\
\hline & TP & FP & Total & TP & FP & Total \\
\hline 1 & 12 & 17 & 29 & 13 & 20 & 33 \\
\hline 2 & 12 & 17 & 29 & 13 & 31 & 44 \\
\hline 3 & 13 & 22 & 35 & 13 & 27 & 40 \\
\hline 4 & 12 & 27 & 39 & 13 & 31 & 44 \\
\hline 5 & 13 & 38 & 51 & 13 & 34 & 47 \\
\hline 6 & 13 & 32 & 45 & 13 & 28 & 41 \\
\hline Average & 12.5 & 25.3 & 37.8 & 13 & 28.5 & 41.5 \\
\hline
\end{tabular}

Data are the numbers of biopsy recommendations for the malignant (true-positive [TP]) and benign (false-positive [FP]) cases. The total numbers of malignant and benign masses are 13 and 38, respectively. 


\section{Mass Detection}

Overall, masses were correctly located in 3D-AUS volumes in $96 \%$ of the readings of the study. Using the automated coregistration, 1 radiologist correctly located the masses on the US images in all 51 cases, 1 radiologist in 50 cases, 1 radiologist in 49 cases, 2 radiologists in 48 cases, and a fifth radiologist in 47 cases. There were a total of 4 cases for which missed registration of the masses on the $3 \mathrm{D}$-AUS images occurred. Of these 4 cases, there were a total of 13 falsepositive detections composed of 6 marks at wrong locations and 7 incorrect negative calls. From the ratings of the second reading, ie, of the correct masses marked on the 3DAUS images by the reference radiologists before the reading, it was observed that the readers recommended on average 2.5 of these 4 cases for biopsy with DBT and 3.5 with combined DBT/3D-AUS. There were 2 cases with negative 3DAUS findings, and each was called negative by all readers.

\section{Survey Questions}

The confidence of each reader to perform simultaneous screening and diagnostic examinations was significantly improved with the addition of 3D-AUS $(P<.05$, Student 2 -tailed paired $t$ test; Table 6 ). Four radiologists perceived combined DBT/3D-AUS as potentially useful in some cases for screening and diagnosis during the same examination; 2 readers expressed a more neutral opinion: The average confidence ratings for the 6 readers were 4.5, 4.8, 4.6, 4.5, 3.1, and 2.1 for the use of DBT alone and 6.3, 7.8, 8.0, 9.4, 7.9 , and 4.5 , respectively, for the use of combined DBT/3D-AUS. The radiologists expressed increased confidence when 3D-AUS was added compared to DBT alone in $80 \%$ of the cases, resulting in an $86 \%$ increase in the overall mean.

\section{Discussion}

Half of all of the readers had sensitivity of $100 \%$ with DBT alone. With the addition of 3D-AUS, the sensitivity for all readers was $100 \%$. The average change in the number of true-positive findings was small, +0.5 true-positive finding per reader, with the addition of 3D-AUS because half of the readers reached sensitivity of $100 \%$ with DBT alone.

Table 4. Changes in Biopsy Recommendations (Changes in BI-RADS Categories From DBT to DBT/3D-AUS)

\begin{tabular}{|c|c|c|c|c|c|c|}
\hline \multirow[b]{2}{*}{ Radiologist } & \multicolumn{2}{|c|}{ Malignant Masses } & \multicolumn{2}{|c|}{ Benign Masses } & \multicolumn{2}{|c|}{ Cysts } \\
\hline & Beneficial & Detrimental & Beneficial & Detrimental & Beneficial & Detrimental \\
\hline 1 & 1 & 0 & 4 & 7 & 2 & 1 \\
\hline 3 & 0 & 0 & 7 & 12 & 6 & 4 \\
\hline 4 & 1 & 0 & 4 & 8 & 2 & 3 \\
\hline 5 & 0 & 0 & 4 & 0 & 4 & 0 \\
\hline Total & 3 & 0 & 27 & 45 & 21 & 13 \\
\hline
\end{tabular}

Changes in BI-RADS categories denote changes from category 1, 2, or 3 (no biopsy) to category 4 or 5 (biopsy recommended). A beneficial change for a malignant mass is a decision based on DBT/3D-AUS to recommend biopsy for the mass that was not recommended for biopsy based on DBT alone. A beneficial change for a benign mass (including the cysts) is a decision based on DBT/3D-AUS not to recommend biopsy for the mass that was recommended for biopsy based on DBT alone.

Table 5. Numbers of Biopsy Recommendations for the Cyst Cases

\begin{tabular}{lcc}
\hline \multirow{2}{*}{ Radiologist } & \multicolumn{2}{c}{ No. of Biopsy Recommendations } \\
\cline { 2 - 3 } & DBT & DBT/3D-AUS \\
\hline 1 & 3 & 2 \\
2 & 4 & 6 \\
3 & 6 & 4 \\
4 & 7 & 8 \\
5 & 12 & 8 \\
6 & 8 & 4 \\
Average & 6.7 & 5.3 \\
\hline
\end{tabular}

The change in biopsy recommendations was not significant for any of the 6 readers ( $P>.13$, McNemar test). The number of cyst cases is 13 .
Table 6. Average Ratings on a 1 to 10 Scale For the Survey Question Concerning the Confidence of Using DBT and Combined DBT/3D-AUS for Screening and Diagnosis After Reading DBT and DBT/3D-AUS Volumes

\begin{tabular}{lcc}
\hline Radiologist & DBT & DBT/3D-AUS \\
\hline 1 & 4.5 & 6.3 \\
2 & 4.8 & 7.8 \\
3 & 4.6 & 8.0 \\
4 & 4.5 & 9.4 \\
5 & 3.1 & 7.9 \\
6 & 2.1 & 4.5 \\
\hline
\end{tabular}

For every reader, the mean ratings for the two modalities were statistically different $(P<.05$, Student 2 -tailed paired $t$ test). 
The specificity decreased with the addition of 3D-AUS, and for one of the readers, it decreased significantly. These results are reasonably consistent with other studies, given the design and small size of this initial study of a new technique. Specifically, in the other studies, when used in addition to mammography, 3D-AUS (and handheld US ${ }^{40}$ ) increased the number of callbacks when used for screening $^{6}$ or increased the number of false-positive findings for mass characterization. ${ }^{17}$

Three readers slightly improved their $\mathrm{A}_{\mathrm{z}}$ with the addition of 3D-AUS. One had a substantial drop. Overall, no significant changes in the average $A_{z}$ across the 6 readers were observed. This result is not unexpected considering the high $\mathrm{A}_{\mathrm{z}}$ value obtained with DBT alone in this study, leaving small room for improvement. In a previous study, it was found that when used as an adjunct to mammography for mass characterization, ${ }^{17}$ 3D-AUS significantly improved the $\mathrm{A}_{\mathrm{z}}$ value from 0.87 (mammography alone) to 0.93 (mammography/3D-AUS), whereas we observed a stable $\mathrm{A}_{\mathrm{z}}$ value of 0.92 for $\mathrm{DBT}$ and combined DBT/3D-AUS.

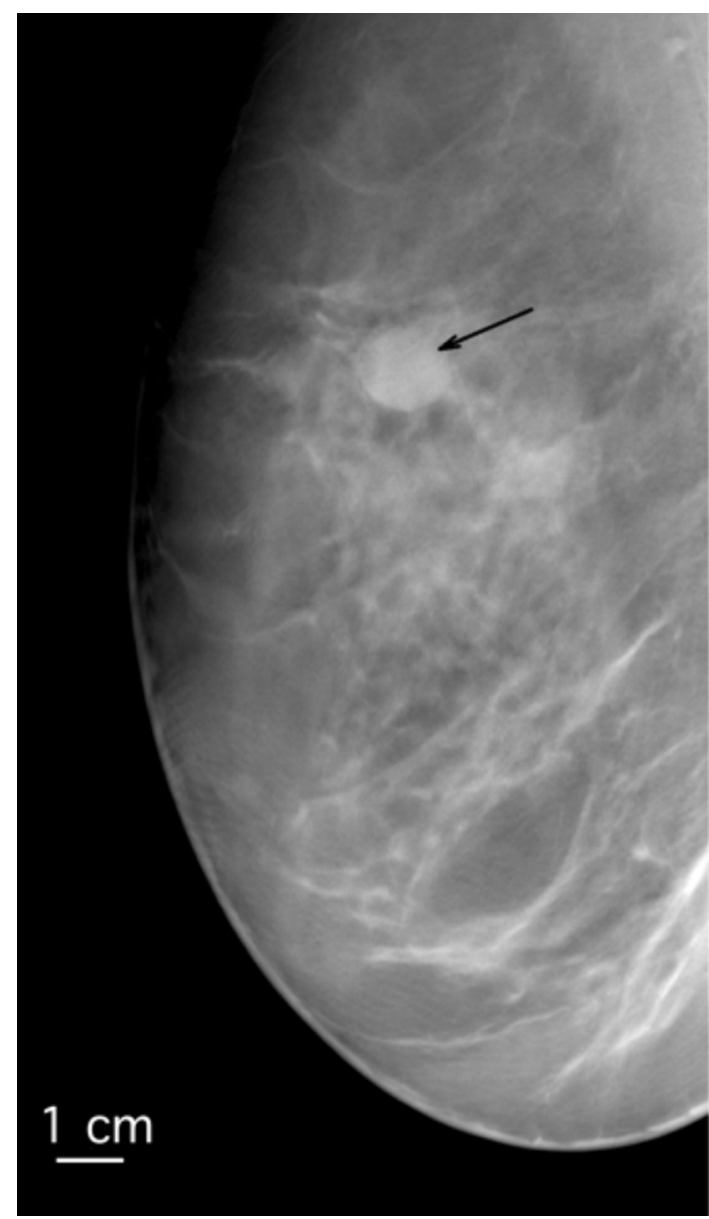

Despite our experience of improved mass visibility on DBT over mammography, ${ }^{21,26,28,30}$ the lack of outstanding results for DBT in much of the literature ${ }^{41}$ and the malignant case that was misdiagnosed in this study by 3 radiologists with DBT alone and was corrected with the addition of 3D-AUS suggest a continuing need for the use of US for mass characterization. The DBT and 3D-AUS images of the case misdiagnosed by 3 radiologists with DBT alone are shown in Figure 2. The tomosynthesis image shows an oval mass with circumscribed margins, features that would lead a radiologist to believe that it would likely be a cyst or a fibroadenoma. The 3D-AUS image shows that the mass is hypoechoic, and has slightly indistinct margins. These features indicate that it is not a simple cyst. If the mass were new or unknown in stability, it would need fine-needle aspiration or biopsy to determine whether it was a complicated cyst, a fibroadenoma, or a malignancy.

Three-dimensional AUS also could be important for delineating complete extension of masses and for imaging masses in dense breasts, as illustrated in Figures 3 and 4.

Figure 2. Sections extracted from mediolateral oblique views of a metaplastic carcinoma in a right breast (left, DBT image; right 3D-AUS image; black arrows point toward the mass) diagnosed as benign on the DBT image by 3 readers. The AUS image is from a plane corresponding to the image plane of the DBT image. The US image is magnified relative to the DBT image.

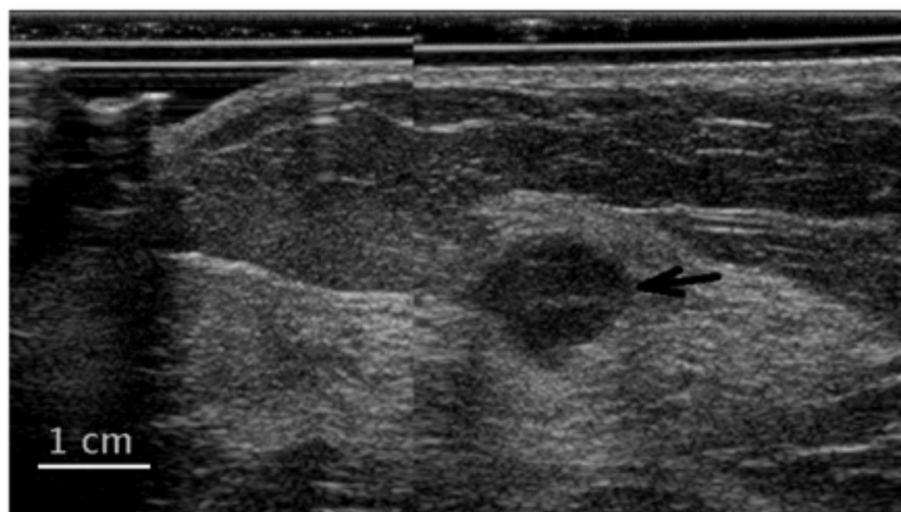


In Figure 3, the 3D-AUS image shows the complete delineation of the mass (large box), which is larger than initially seen on DBT and confirmed by pathologic examination after mass excision. In Figure 4, the DBT image and also the mammogram were so dense that the mass was only distinguishable because of the surrounding fat on three sides, whereas the mass is clearly evident in the 3D-AUS image.

Some false-positive results may be explained by suboptimal quality of some of the 3D-AUS images when scanned from only one side of the breast, as in this study. Breast US imaging using mammographic compression may cause reverberation artifacts in cysts, as illustrated in Figure 5. Reverberation artifacts are observed inside the mass on the 3-AUS image, probably due to reverberation involving the solid compression paddle and hyperechoic structures located above the cyst. The orientations of these structures in mammographic compression geometry are more perpendicular to the direction of propagation of the US beam than they would be for a handheld US scan, resulting in stronger echoes. Limitations in image quality may also result from an increased distance between the US probe and the target mass in mammographic compression geometry compared to handheld US imaging and from scattering by tissue structures, ${ }^{42}$ particularly structures not as flattened as they would be on optimized handheld US
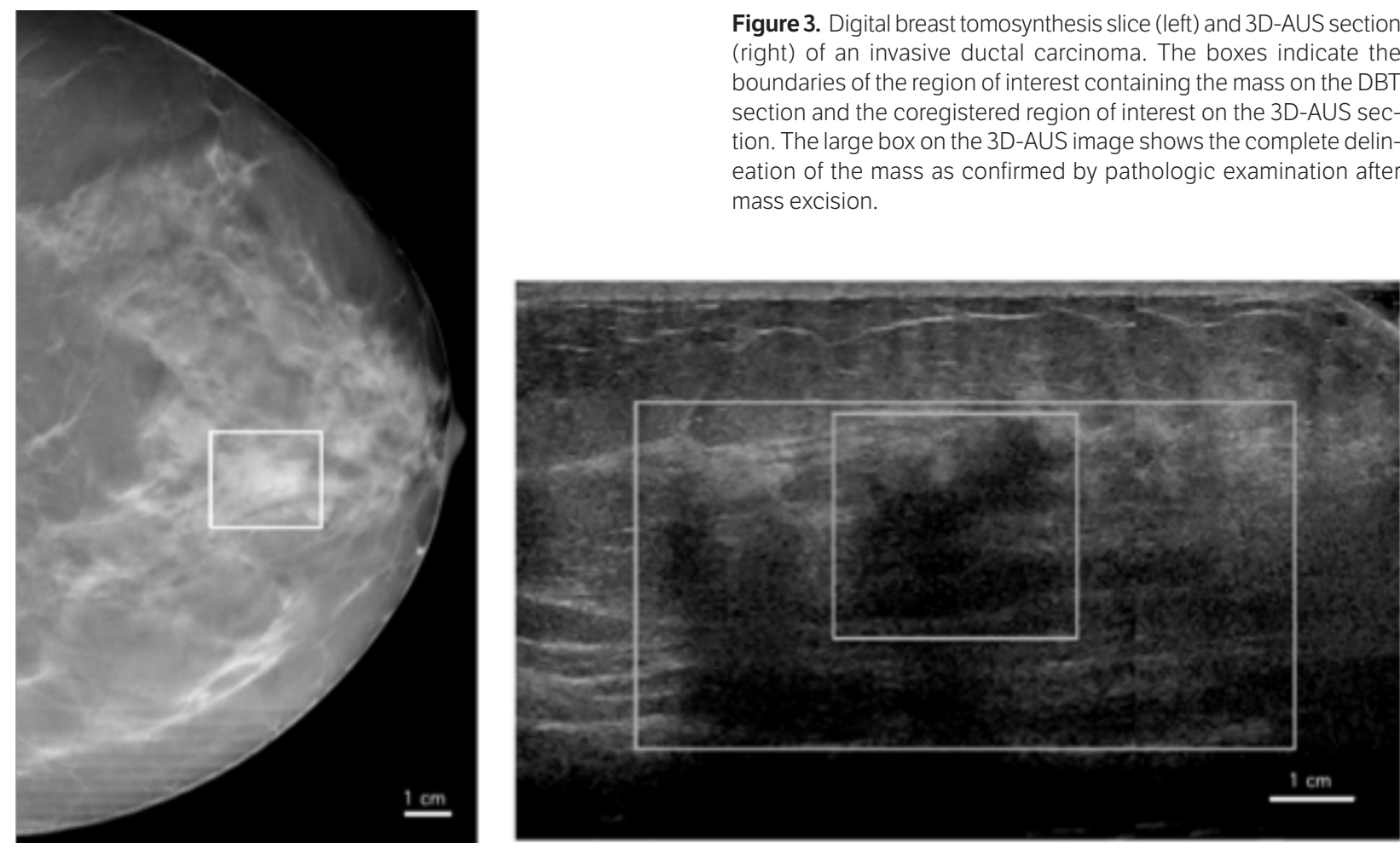

images, or from the presence of air bubbles in the coupling media, $7,9,12,13$ especially in the pariareolar and lateral areas of the breast. ${ }^{9}$ In addition, in 3D-AUS imaging as performed here, there is limited coverage of the breast, responsible for the exclusion of $25 \%$ of the research cases.

For almost three-fourths of these excluded cases, the mass was located too far posterior, an area of the breast difficult to image with US when the transducer beam is oriented in a craniocaudal direction while the breast is under mammographic compression. Improvements should come from the use of multiple 3D-AUS views. We plan systematic acquisitions of two 3D-AUS views in our future protocol, providing two volumes coregistered with two DBT volumes, improving the coverage and field of view for the US imaging and potentially also allowing discrimination of acoustic shadowing artifacts. For the cases excluded because the mass was located at the edge of the breast, the issue usually arose from ineffective coupling of the transducer/gel to the skin. We recently developed greatly improved techniques for gel positioning and maintenance in the large gaps between the transducer/compression paddle surface and the edge of the breast.

Substantial improvements in 3D-AUS image quality could also be obtained with the use of the compound imaging mode, speckle reduction, better acoustic coupling via

Figure 3. Digital breast tomosynthesis slice (left) and 3D-AUS section (right) of an invasive ductal carcinoma. The boxes indicate the boundaries of the region of interest containing the mass on the DBT section and the coregistered region of interest on the 3D-AUS section. The large box on the 3D-AUS image shows the complete delineation of the mass as confirmed by pathologic examination after mass excision. 
new coupling gel control and application methods, ${ }^{9}$ a new mesh paddle, ${ }^{35}$ scanning from both sides of the breast ${ }^{43}$ with transducers shaped to allow imaging close to the chest wall, other enhanced scanning and acquisition techniques, ${ }^{44,45}$ and multiple $3 \mathrm{D}$-AUS views.

In our study, automatic registration was perceived as very useful by the radiologists and resulted in the correct identification of masses in $96 \%$ of the readings. Missed registrations were concentrated in 4 cases, for which 3D-AUS volumes were found very difficult to interpret because of a variety of limitations: the depth and very small size of the masses or an offset in the registration probably caused by an inadvertent posterior pullback of the breast by the patient between the DBT and 3D-AUS scans.

Most of the radiologists perceived combined DBT/ 3D-AUS as potentially useful for screening and diagnosis of some cases during the same examination. The question of the usefulness of screening with US is still debated, but several major studies reported improved incremental cancer detection with handheld US $S^{1,40,46,47}$ and AUS. ${ }^{6,18}$ Similar BI-RADS classification of cysts between handheld US and 3D-AUS has also been reported. ${ }^{7}$ If the full potential of US to differentiate simple cysts from solid masses can be confirmed in a system combining it with DBT, as reported here, that system could be useful for both screening and diagnostic workup of many cases in a single examination. This factor is especially important because simple cysts constitute $25 \%$ to $27 \%$ of all palpable or mammographically detected lesions in the general diagnostic population. ${ }^{46,48}$ This role of 3D-AUS should be particularly efficacious and could therefore improve the potential ${ }^{23}$ of DBT in screening and diagnostic breast imaging practices.

One commercial DBT system recently received US Food and Drug Administration approval, and the role of tomosynthesis in breast imaging is likely to increase rapidly. Three-dimensional AUS is compatible with the design of mammography and tomosynthesis systems and could be implemented using clinical US scanners, at the cost of the installation of US-compatible compression paddles with one or two motorized transducer carriages and controls. The reading time of combined system data could be reduced by the use of computer-aided detection ${ }^{49-51}$ and automatic registration $^{52}$ to reach an acceptable standard for clinical practice. The use of computer-aided detection could also improve mass characterization, ${ }^{17,53}$ particularly given coregistered US and DBT images.

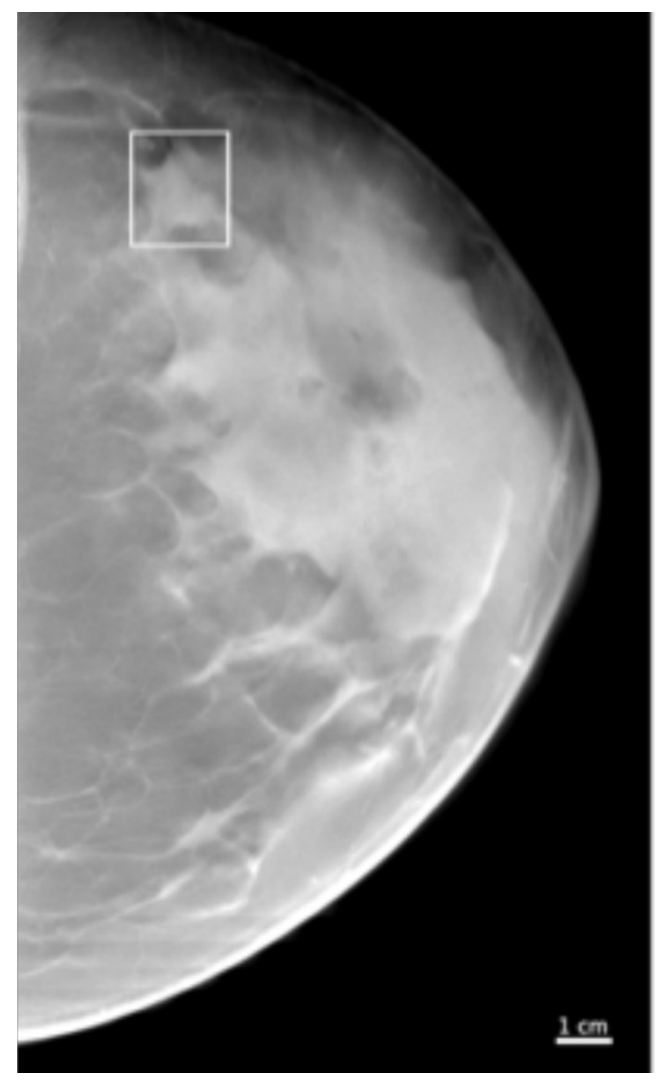

Figure 4. Digital breast tomosynthesis slice (left) and 3D-AUS section (right) of an invasive ductal carcinoma. The boxes indicate the boundaries of the region of interest containing the mass on the DBT section and the coregistered region of interest on the 3D-AUS section.

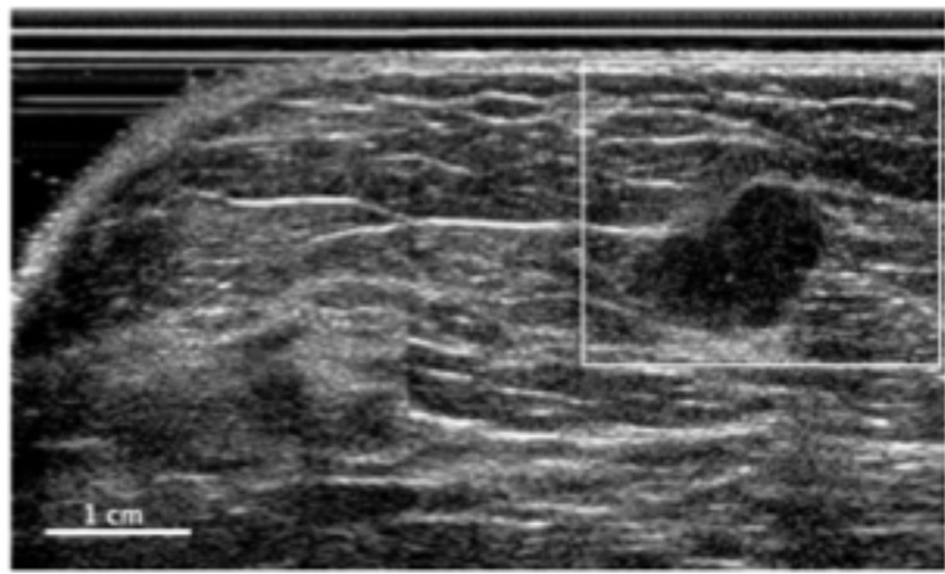


The prevalence of cancer in our study population was higher than that in a diagnostic population in clinical practice, but it has been reported that prevalence between $2 \%$ and $28 \%$ would not significantly affect the outcomes of an ROC study. ${ }^{54}$

Our study had a number of limitations. First, the inclusion criteria could have introduced an unintended bias toward tomosynthesis. This study being a characterization study, only cases for which the mass was seen on DBT image and was in the field of view of the US image were used. Second, because biopsy results were used as truth, all of the masses that were analyzed had been recommended for biopsy after mammographic and handheld US imaging. Thus, masses present in a diagnostic population with benign diagnoses after mammographic and handheld US imaging were not included. Third, the study involved only 51 patients and 6 readers, thereby limiting our ability to

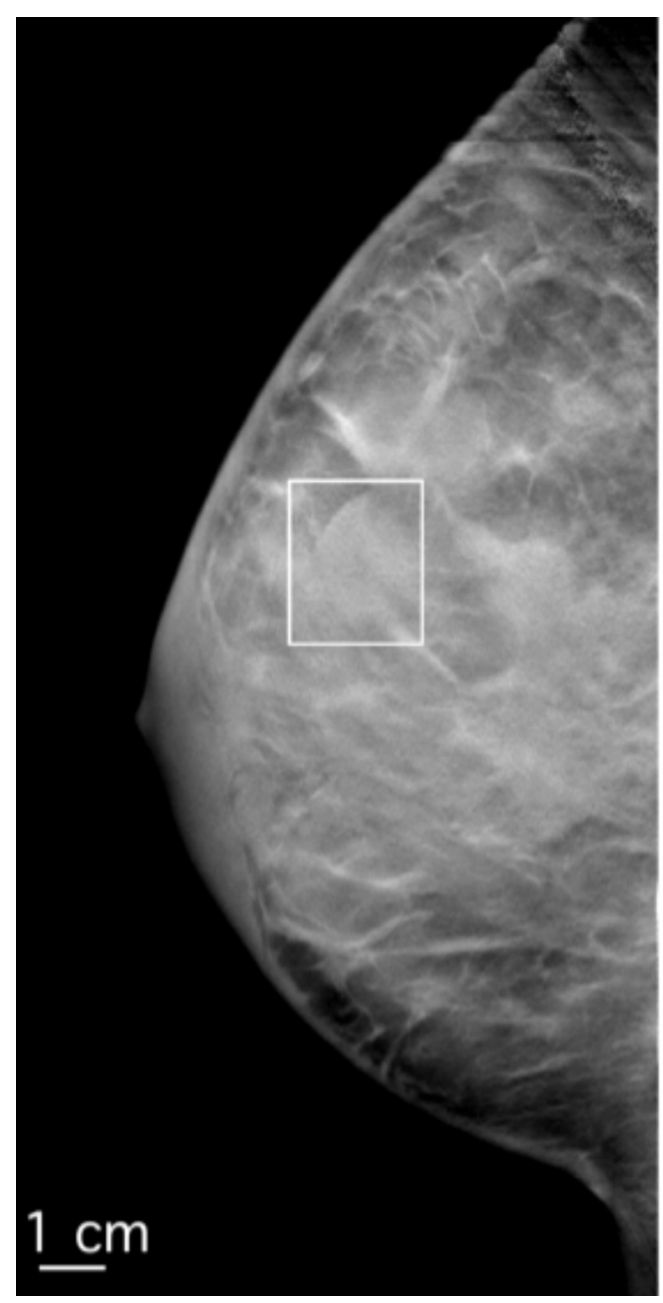

assess small differences between the two modalities. Fourth, all observers from our study were experienced radiologists in breast imaging, with some experience in DBT reading. Thus, our results may not generalize to radiologists with different experience.

In summary, we have reported one of the early studies of US imaging with tomosynthesis, with may trigger interest for AUS in this geometry and show some directions for further improvements. In this pilot reader study, the characterization of masses by the radiologists was not statistically improved by the addition of 3D-AUS. However, we tentatively believe that these preliminary results are encouraging for the following reasons: (1) the addition of 3D-AUS led to the diagnosis of 1 additional malignant case by half of the readers; and (2) a trend toward a decrease in the number of biopsy recommendations was observed for the cyst cases. The realized 3D-AUS image quality and

Figure 5. Digital breast tomosynthesis slice (left) and 3D-AUS section (right) of a cyst. The boxes indicate the boundary of the region of interest containing the mass on the DBT slice and the coregistered region of interest on the 3D-AUS section. Reverberation artifacts can be observed inside the mass on the 3D-AUS image.

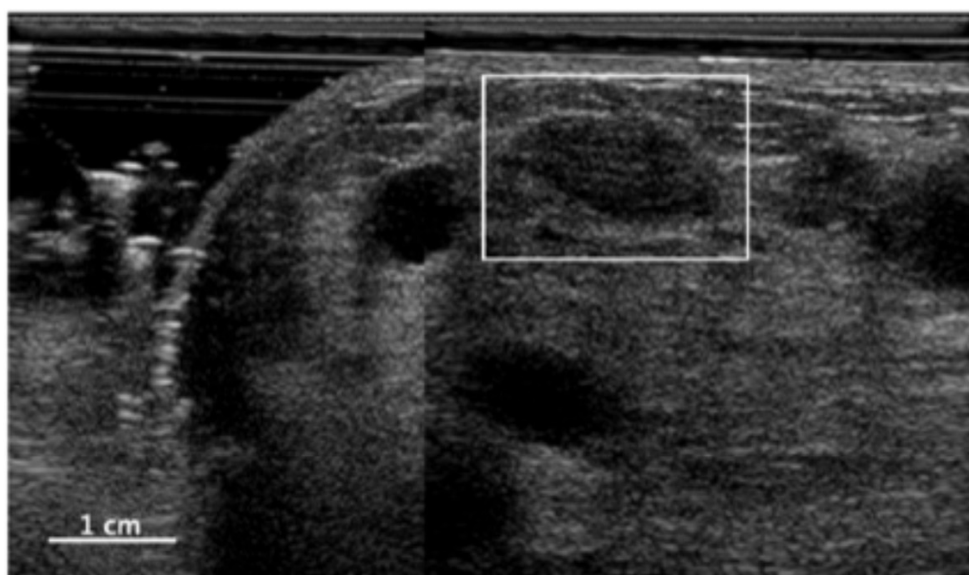


breast coverage may be limiting factors of the prototype used, especially for mass identification, but substantial improvements are possible. Most of the readers indicated increased confidence in potentially performing simultaneous screening and diagnostic examinations with a combined DBT/3D-AUS system. Assessing the performance of a revised combined system in an enhanced screening population will be the objective of future studies.

\section{References}

1. Kolb TM, Lichy J, Newhouse JH. Comparison of the performance of screening mammography, physical examination, and breast US and evaluation of factors that influence them: an analysis of 27,825 patient evaluations. Radiology 2002; 225:165-175.

2. Jackson VP. The role of US in breast imaging. Radiology 1990; 177:305311.

3. Skaane $P$, Engedal K. Analysis of sonographic features in the differentiation of fibroadenoma and invasive ductal carcinoma.AJRAm J Roentgenol 1998; 170:109-114.

4. Stavros AT, Thickman D, Rapp CL, Dennis MA, Parker SH, Sisney GA. Solid breast nodules: use of sonography to distinguish between benign and malignant lesions. Radiology 1995; 196:123-134.

5. Taylor KJ, Merritt C, Piccoli C, et al. Ultrasound as a complement to mammography and breast examination to characterize breast masses. Ultrasound Med Biol 2002; 28:19-26.

6. Kelly KM, Dean J, Comulada WS, Lee SJ. Breast cancer detection using automated whole breast ultrasound and mammography in radiographically dense breasts. Eur Radiol 2010; 20:734-742.

7. Wenkel E, Heckmann M, Heinrich M, et al. Automated breast ultrasound: lesion detection and BI-RADS classification — a pilot study. Rofo 2008; 180:804-808.

8. Goodsitt MM, Chan HP, Hadjiiski LM, et al. Automated registration of volumes of interest for a combined $\mathrm{x}$-ray tomosynthesis and ultrasound breast imaging system. Lect Notes Comput Sci 2008; 5116:463-468.

9. Li J, Goodsitt MM, Padilla F, et al. Effect of a gel retainment dam on automated ultrasound coverage in a dual-modality breast imaging system. JUltrasound Med 2010; 29:1075-1081.

10. Sinha SP, Goodsitt MM, Roubidoux MA, et al. Automated ultrasound scanning on a dual-modality breast imaging system: coverage and motion issues and solutions. J Ultrasound Med 2007; 26:645-655.

11. Sinha SP, Roubidoux MA, Helvie MA, et al. Multi-modality 3D breast imaging with $\mathrm{x}$-ray tomosynthesis and automated ultrasound. Conf Proc IEEE Eng Med Biol Soc 2007; 2007:1335-1338.

12. Kotsianos-Hermle D, Hiltawsky KM, Wirth S, Fischer T, Friese K, Reiser M. Analysis of 107 breast lesions with automated 3D ultrasound and comparison with mammography and manual ultrasound. Eur J Radiol 2009; $71: 109-115$

13. Kotsianos-Hermle D, Wirth S, Fischer T, Hiltawsky KM, Reiser M. First clinical use of a standardized three-dimensional ultrasound for breast imaging. Eur J Radiol 2009; 71:102-108.
14. Richter K, Prihoda H, Heywang-Köbrunner SH, Hamm B. Description and first clinical use of a new system for combined mammography and automated clinical amplitude/velocity reconstructive imaging breast sonography. Invest Radiol 1997; 32:19-28.

15. Richter K, Willrodt RG, Opri F, Heywang-Köbrunner SH. Differentiation of breast lesions by measurements under craniocaudal and lateromedial compression using a new sonographic method. Invest Radiol 1996; 31:401-414.

16. Callahan KS, Borup DT, Johnson SA, et al. Transmission breast ultrasound imaging: representative case studies of speed of sound and attenuation of sound computed tomographic images. Am J Clin Oncol 2007; 30:458-459.

17. Sahiner B, Chan HP, Hadjiiski LM, et al. Multi-modality CADx: ROC study of the effect on radiologists' accuracy in characterizing breast masses on mammograms and 3D ultrasound images. Acad Radiol 2009; 16:810818.

18. Kelly KM, Dean J, Lee SJ, Comulada WS. Breast cancer detection: radiologists' performance using mammography with and without automated whole-breast ultrasound. Eur Radiol 2010; 20:2557-2564.

19. Conway WF, Hayes CW, Brewer WH. Occult breast masses: use of a mammographic localizing grid for US evaluation. Radiology 1991; 181:143-146

20. BergWA,Woel BS. Mammographic-sonographic correlation. Ultrasound Clin 2007; 1:567-591.

21. Helvie MA. Digital mammography imaging: breast tomosynthesis and advanced applications. Radiol Clin North Am 2010; 48:917-929.

22. Gennaro G, Baldan E, Bezzon E, et al. Clinical performance of digital breast tomosynthesis versus full-field digital mammography: preliminary results. Lect Notes Comput Sci 2008; 5116:477-482

23. Good WF, Abrams GS, Catullo VJ, et al. Digital breast tomosynthesis: a pilot observer study. AJR Am J Roentgenol 2008; 190:865-869.

24. Poplack SP, Tosteson TD, Kogel CA, Nagy HM. Digital breast tomosynthesis: initial experience in 98 women with abnormal digital screening mammography. AJR Am J Roentgenol 2007; 189:616-623.

25. Andersson I, Ikeda DM, Zackrisson S, et al. Breast tomosynthesis and digital mammography: a comparison of breast cancer visibility and BIRADS classification in a population of cancers with subtle mammographic findings. Eur Radiol 2008; 18:2817-2825.

26. Helvie MA, Roubidoux MA, Zhang Y, et al. Tomosynthesis mammography versus conventional mammography: lesion detection and reader preference - initial experience. Paper presented at: Radiological Society of North America 92nd Scientific Assembly and Annual Meeting; November 26-December 1, 2006; Chicago, IL.

27. LoJ, Durham N, Orman J, et al. Breast tomosynthesis: initial clinical experience with 100 human subjects. Paper presented at: Radiological Society of North America 92nd Scientific Assembly and Annual Meeting; November 26-December 1, 2006; Chicago, IL.

28. Helvie MA, Roubidoux MA, Hadjiiski LM, et al. Research digital tomosynthesis mammography: detection of $\mathrm{T} 1$ invasive breast carcinomas not diagnosed by conventional breast imaging or physical exam. Paper presented at: Radiological Society of North America 94th Scientific 
Assembly and Annual Meeting; November 30-December 5, 2008; Chicago, IL.

29. Helvie MA, Hadjiiski LM, Goodsitt MM, et al. Characterization of benign and malignant breast masses by digital breast tomosynthesis mammography. Paper presented at: Radiological Society of North America 94th Scientific Assembly and Annual Meeting; November 30-December 5, 2008; Chicago, IL.

30. Helvie MA, Roubidoux MA, Hadjiiski LM, et al. Tomosynthesis mammography vs conventional mammography: comparison of breast mass detection and characterization. Paper presented at: Radiological Society of North America 93rd Scientific Assembly and Annual Meeting; November 25-30, 2007; Chicago, IL.

31. Teertstra HJ, Loo CE, van den Bosch MAAJ, et al. Breast tomosynthesis in clinical practice: initial results. Eur Radiol 2010; 20:16-24.

32. Zhang Y, Chan HP, Sahiner B, et al. A comparative study of limited-angle cone-beam reconstruction methods for breast tomosynthesis. Med Phys 2006; 33:3781-3795.

33. Booi RC, Krücker JF, Goodsitt MM, et al. Evaluating thin compression paddles for mammographically compatible ultrasound. Ultrasound Med Biol 2007; 33:472-482.

34. Kapur A, Carson PL, Eberhard J, et al. Combination of digital mammography with semi-automated 3D breast ultrasound. Technol Cancer Res Treat 2004; 3:325-334

35. Blane CE, Goodsitt MM, Grimm JC, et al. New compression paddle for wire localization in mammography. Acad Radiol 2010; 17:142-145.

36. Wagner RF, Metz CE, Campbell G. Assessment of medical imaging systems and computer aids: a tutorial review. Acad Radiol 2007; 14:723-748.

37. Metz CE. Some practical issues of experimental design and data analysis in radiological ROC studies. Invest Radiol 1989; 24:234-245.

38. Dorfman DD, Berbaum KS, Metz CE. Receiver operating characteristic rating analysis: generalization to the population of readers and patients with the jackknife method. Invest Radiol 1992; 27:723-731.

39. Pesce LL, Metz CE. Reliable and computationally efficient maximumlikelihood estimation of "proper" binormal ROC curves. Acad Radiol 2007; 14:814-829.

40. Berg WA, Blume JD, Cormack JB, et al. Combined screening with ultrasound and mammography vs mammography alone in women at elevated risk of breast cancer. JAMA 2008; 299:2151-2163.

41. Diekmann F, Bick U. Breast tomosynthesis. Semin Ultrasound CT MR $2011 ; 32: 281-287$

42. Hovanessian Larsen L, Wallman M, Johnson M, et al. Can automated whole breast ultrasound replace hand-held ultrasound in a busy diagnostic breast center? Paper presented at: Radiological Society of North America 94th Scientific Assembly and Annual Meeting; November 30December 5, 2008; Chicago, IL.

43. Carson PL, Fouzaan Z, van der Spek S, et al. Dual sided automated ultrasound system in the mammographic geometry. Paper presented at: IEEE International Ultrasonics Symposium; October 18-21,2011; Orlando, FL.

44. Tozaki M, Isobe S, Yamaguchi M, et al. Optimal scanning technique to cover the whole breast using an automated breast volume scanner. Jpn J Radiol 2010; 28:325-328
45. Carson PL, Wang B, LeCarpentier GL, et al. Local compression in automated breast ultrasound in the mammographic geometry. Paper presented at: 2010 IEEE International Ultrasonics Symposium; October 11-14, 2010; San Diego, CA.

46. Kolb TM, Lichy J, Newhouse JH. Occult cancer in women with dense breasts: detection with screening US — diagnostic yield and tumor characteristics. Radiology 1998; 207:191-199.

47. Berg WA. Rationale for a trial of screening breast ultrasound: American College of Radiology Imaging Network (ACRIN) 6666. AJR Am J Roentgenol 2003; 180:1225-1228.

48. Hilton SV, Leopold GR, Olson LK, Willson SA. Real-time breast sonography: application in 300 consecutive patients. AJR Am J Roentgenol 1986; 147:479-486.

49. Chang RF, Chang-Chien KC, Takada E, et al. Rapid image stitching and computer-aided detection for multipass automated breast ultrasound. Med Phys 2010; 37:2063-2073.

50. Ikedo Y, Fukuoka D, Hara T, et al. Computerized mass detection in whole breast ultrasound images: reduction of false positives using bilateral subtraction technique [abstract]. In: Medical Imaging 2007: Computer-Aided Diagnosis, Proceedings of SPIE. Bellingham, WA: SPIE; 2007:65142007.

51. Chan HP, Wei J, Zhang YH, et al. Computer-aided detection of masses in digital tomosynthesis mammography: comparison of three approaches. Med Phys 2008; 35:4087-4095.

52. Sinha SP, Roubidoux MA, Goodsitt MM, Hadjiyski LM, Thomenius KE Carson PL. Time-efficient mass localization on automated breast ultrasound with dual-modality information [abstract]. JUltrasound Med 2009; 28(suppl):S40.

53. Chan HP, Wu YT, Sahiner B, et al. Characterization of masses in digital breast tomosynthesis: comparison of machine learning in projection views and reconstructed slices. Med Phys 2010; 37:3576-3586.

54. GurD, Rockette HE, Armfield DR, et al. Prevalence effect in a laboratory environment. Radiology 2003; 228:10-14. 